\section{Effect of phenolic compounds and osmotic stress on the expression of penicillin biosynthetic genes from Penicillium chrysogenum var. halophenolicum strain}

\author{
Sumaya Ferreira Guedes, ${ }^{1,2}$ \\ Ana Lúcia Leitão'
}

'Departamento de Ciências e Tecnologia da Biomassa, Faculdade de Ciências e Tecnologia, Universidade Nova de Lisboa, Campus de Caparica; ${ }^{2}$ Instituto de Química, Universidade Estadual de Campinas (UNICAMP), Portugal

\section{Abstract}

Phenol and phenolic compounds are aromatic pollutants that inhibit biological treatment of wastewaters. Penicillium chrysogenum var. halophenolicum is a halotolerant fungus that previously showed the ability to degrade phenol and resorcinol in high salinity conditions. The presence of the penicillin biosynthetic cluster in $P$. chrysogenum var. halophenolicum was recently described. In this article, we examined the expression of $p c b A B, p c b C$ and $p e n D E$, genes responsible for $\delta$-(L- $\alpha$-aminoadipyl)-L-cysteinyl-D-valine synthetase, isopenicillin $\mathrm{N}$ synthase and isopenicillin $\mathrm{N}$ acyltransferase activities, respectively, in $P$. chrysogenum var. halophenolicum. A quantitative PCR (qPCR) approach was used to determine how these genes were expressed in media with $2 \%$ and $5.9 \% \mathrm{NaCl}$ supplemented with phenol, catechol, hydroquinone and resorcinol as the sole carbon source. The effect of salt on the capability of $P$. chrysogenum var. halophenolicum to degrade aromatic compounds was measured using HPLC. qPCR analysis of RNA extracted from $P$. chrysogenum var. halophenolicum indicated that the expression levels of $p c b A B, p c b C$ and $p e n D E$ decreased in high saline concentrations compared to the levels expressed in media with glucose. High concentrations of salt significantly repress the expression of $p c b A B$ and $p e n D E$. The $p c b C$ gene was expressed differentially in catechol containing medium. There was no evident relationship between the expression levels of penicillin biosynthetic genes and yields of penicillin. Meanwhile, the presence of phenol and phenolic compounds seems to positively influence the antibiotic production; high concentrations of salt stimulated penicillin production. These results support the hypothesis that phenol, phenolic compounds and high concentrations of salt could act like a stress factor for $P$. chrysogenum var. halophenolicum resulting in higher yields of $\beta$-lactam antibiotic production.

\section{Introduction}

The production of penicillin by filamentous fungi has been considered as the beginning of modern pharmaceutical industry. In 2002, the total world market of $\beta$-lactam antibiotics was estimated to be approximately 15 billion US dollars; penicillin accounts for sales of approximately 5 billion US dollars. ${ }^{1}$

Penicillium chrysogenum is an ascomycete fungus able to synthesize penicillin with both aromatic and non-aromatic side chains. ${ }^{2}$ The genes for the synthesis of secondary metabolites, like antibiotics, are frequently arranged in clusters. ${ }^{3}$ In the case of penicillin biosynthesis, the cluster is constituted by three genes, $p c b A B, p c b C$ and $p e n D E$, comprising a $15-\mathrm{kb}$ DNA region (Figure 1). While in Fleming's isolate this region contains only a single copy of the cluster, in industrial penicillin overproducer strains this segment is amplified in tandem repeats. For example, in the AS-P-78 strain, an overproducer strain obtained at Antibioticos SA (Léon, Spain), the amplified region is present in five or six copies. ${ }^{4}$ The $p c b A B$ and $p c b C$ genes are expressed from a 1.16-kb bidirectional promoter region in opposite directions and encode $\delta$-(L- $\alpha$-aminoadipyl)-L-cysteinylD-valine synthetase and isopenicillin $\mathrm{N}$ synthase, respectively. ${ }^{5,6}$ The third gene, penDE, is responsible for the activity of isopenicillin $\mathrm{N}$ acyltransferase. ${ }^{6}$ In the penicillin biosynthesis pathway, the first step is the non-ribosomal condensation of the three precursor amino acids, L- $\alpha$-aminoadipic acid, L-cystein, and Lvaline, by the enzyme synthetase that was found to be associated with membranes or small organelles. ${ }^{5,6}$ The product of this reaction is the tripeptide $\delta(\mathrm{L}$ - $\alpha$-aminoadipyl $)$-L-cysteinyl-D-valine (ACV), which is cyclized to isopenicillin N (IPN) by the activity of cytosolic enzyme, the isopenicillin synthase.$^{7,8}$ The isopenicillin $\mathrm{N}$ is later modified by the enzyme isopenicillin acyltransferase that was located in organelles to form penicillin G (Figure 1). ${ }^{8}$

It is well documented that the expression of the three genes, $p c b A B, p c b C$ and $p e n D E$, is controlled by a complex regulatory network, comprising both nutritional and developmental factors. ${ }^{5,9}$ For example, glucose and sucrose negatively regulated the penicillin biosynthesis in $P$. chrysogenum, maltose, galactose and fructose had a less negative effect. ${ }^{9}$ Indeed, glucose strongly represses the transcription of $p c b A B$ and $p c b C$ in $P$. chrysogenum; in opposition to that the gene $p c b C$ is expressed in media with lactose..$^{10}$

Phenol, catechol, hydroquinone and resorci-
Correspondence: Ana Lúcia Leitão, Departamento de Ciências e Tecnologia da Biomassa, Faculdade de Ciências e Tecnologia, Universidade Nova de Lisboa, Campus de Caparica, 2829-516 Caparica, Portugal.

Tel. +351.212 .948 .543 - Fax: +351.212 .948 .543 .

E-mail: aldı@fct.unl.pt

Key words: Penicillium chrysogenum, penicillin biosynthesis cluster, antibiotic production, phenol and phenolic compounds.

Contributions: SFG, performed engineering experiments with phenol, catechol, hydroquinone and resorcinol, as well as antibiotic estimation. ALL, was responsible for this work, performed the quantification of phenol and phenolic compounds by HPLC and qPCR experiments, and elaborated the present paper

Received for publication: 9 November 2011.

Revision received: 9 January 2012.

Accepted for publication: 9 January 2012.

This work is licensed under a Creative Commons Attribution NonCommercial 3.0 License (CC BYNC 3.0).

(C)Copyright S. Ferreira Guedes and A.L. Leitão, 2012 Licensee PAGEPress srl, Italy

Journal of Xenobiotics 2012; 2:e2

doi:10.4081/xeno.2012.e2

nol are xenobiotic compounds present in many industrial wastewaters that can be used by several bacteria and fungi; however a very limited number of these microorganisms have the ability to utilize all of them, particularly under saline conditions. ${ }^{11-14}$ Recently, our group described the characterization of a halotolerant Penicillium chrysogenum strain isolated from a salt-mine and named as Penicillium chrysogenum var. halophenolicum. ${ }^{15}$ The presence of the full penicillin biosynthetic gene cluster ( $p c b A B, p c b C$ and $p e n D E$ genes) and the biosynthesis of penicillin have been demonstrated in the Penicillium chrysogenum var. halophenolicum strain by Southern hybridizations. ${ }^{15}$ Penicillium chrysogenum var. halophenolicum can metabolize up to $300 \mathrm{mg} / \mathrm{L}$ of phenol and resorcinol at $5.9 \%(\mathrm{w} / \mathrm{v})$ of $\mathrm{NaCl}$ concentration. ${ }^{16,17}$ However, the effects of phenolic compounds and sodium chloride on the genomic output of the penicillin biosynthesis cluster in Penicillium chrysogenum var. halophenolicum remain unknown. In the present work, we aimed to study the effect of phenol, catechol, hydroquinone and resorcinol on the expression of penicillin genes cluster of $P$. chrysogenum var. halophenolicum, when used as the sole carbon source. The effect of these compounds was also investigated at two different saline concentrations $(5.9 \%$ and $2 \%$ of $\mathrm{NaCl}$, under osmotic and non-osmotic stress 
condition, respectively), giving an insight into how high salinities affect the expression levels of penicillin G. Therefore, one of the purposes of this study was to obtain more information on the relationship between primary and secondary metabolism of $P$. chrysogenum var. halophenolicum during the biodegradation of phenolic compounds.

\section{Materials and Methods}

\section{Strains}

P. chrysogenum var. halophenolicum was used throughout this study; this strain was isolated from a salt mine in Algarve, Portugal, and has been previously characterized. ${ }^{17}$

\section{Chemicals}

The phenol, catechol, hydroquinone and resorcinol used in this study were of chromatographic grade (purity $\geq 99 \%$ ), and were obtained from Sigma-Aldrich (St. Louis, USA). HPLC grade acetonitrile was obtained from Lab-Scan (Dublin, Ireland). All other reagents are of analytical-reagent grade and were obtained from Riedel-de Haën (Seelze, Germany). Water purified by a Mili-Q system was used in all the experiments and nutrient agar (NA) was purchased from Difco (Detroit, USA).

\section{Culture conditions}

P. chrysogenum var. halophenolicum was maintained at $4{ }^{\circ} \mathrm{C}$ on nutrient agar plates with $5.9 \%(\mathrm{w} / \mathrm{v}) \mathrm{NaCl}$. Precultures of cells were routinely aerobically cultivated (160 rpm in an INNOVA 4000 Incubator (New Brunswick Scientific, New Jersey, USA) in $100 \mathrm{~mL}$ of complex medium (MC: glucose $30 \mathrm{~g} / \mathrm{L} ; \mathrm{NaNO}_{3} 3.0$ $\mathrm{g} / \mathrm{L} ; \mathrm{MgSO}_{4} .7 \mathrm{H}_{2} \mathrm{O} 0.5 \mathrm{~g} / \mathrm{L} ; \mathrm{NH}_{4} \mathrm{Fe}\left(\mathrm{SO}_{4}\right)_{2} .12 \mathrm{H}_{2} \mathrm{O}$ $10 \mathrm{mg} / \mathrm{L} ; \mathrm{K}_{2} \mathrm{HPO}_{4}, 1.0 \mathrm{~g} / \mathrm{L}$; yeast extract $5.0 \mathrm{~g} / \mathrm{L}$; $\mathrm{NaCl} 58.5 \mathrm{~g} / \mathrm{L}$ or $20 \mathrm{~g} / \mathrm{L} ; \mathrm{pH} 5.6$ ).

To investigate the use of phenolic compounds and $\beta$-lactam antibiotic production, the strain was cultivated in 500 -mL flasks containing $100 \mathrm{~mL}$ of complex medium MC during 68 h. Cells were collected by centrifugation and washed in $0.85 \%(\mathrm{w} / \mathrm{v})$ of $\mathrm{NaCl}$ solution prepared in mili-Q water. A $10 \%$ of the preinoculum $(5.0 \mathrm{~mL})$ was inoculated in $45 \mathrm{~mL}$ of min-

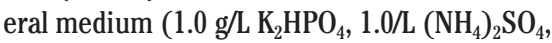
$200 \mathrm{mg} / \mathrm{L} \mathrm{MgSO}_{4} \cdot 7 \mathrm{H}_{2} \mathrm{O}, 33 \mathrm{mg} / \mathrm{L} \mathrm{FeCl}_{3} \cdot 6 \mathrm{H}_{2} \mathrm{O}$, $100 \mathrm{mg} / \mathrm{L} \mathrm{CaCl}, 58.5 \mathrm{~g} / \mathrm{L}$ or $20 \mathrm{~g} / \mathrm{L} \mathrm{NaCl}$; final $\mathrm{pH}$ was adjusted to $5.6-5.8$ with $5 \mathrm{~mol} / \mathrm{L} \mathrm{HCl}$ ), with phenol or catechol or hydroquinone or resorcinol or glucose (final concentration of $2.67 \mathrm{mM}$ ). After $45 \mathrm{~h}$ and $68 \mathrm{~h}$ of culture, cells were harvested and $1.0 \mathrm{~mL}$ of supernatant was maintained at $-60^{\circ} \mathrm{C}$ for antibiotic and aromatic compound determinations. The cells were washed with PBS (pH 7) and $1.0 \mathrm{~mL}$ of Trizol reagent was added to $0.5 \mathrm{~mL}$ of cells before analysis of gene expression by qPCR.

\section{Quantification of gene expression by qPCR}

Total RNA was extracted by using a modified protocol that combined a Trizol reagent extraction (Stratagene, USA) together with column purification. Briefly, fungal mycelia harvested under several growing conditions were homogeneized with Trizol, and extracted once with chloroform following the manufacturer's instructions. The aqueous phase was mixed with 1.5 volumes of absolute ethanol and loaded onto an RNAeasy mini-spin column for purification (Qiagen, USA). Columns were washed and eluted following the manufacturer's instructions. RNA was quantified by spectrophotometric absorption in a Nanodrop 1000 spectrophotometer (Thermo Scientific, USA) and its quality verified by microfluidics analysis with an Agilent Bioanalyzer 2100 (Agilent, USA).

First strand cDNA was synthesized by reverse transcriptase (Superscript II, Invitrogen, USA) from total RNA using oligo(dT) $)_{17}$ primer using $250 \mathrm{ng}$ of total RNA per reaction.

The first strand cDNA was used as a template for the quantification of the relative expression levels of $p c b A B, p c b C$ and $p e n D E$ by qPCR analysis with an ABI Prism 7000 sequence detection system (Applied

A

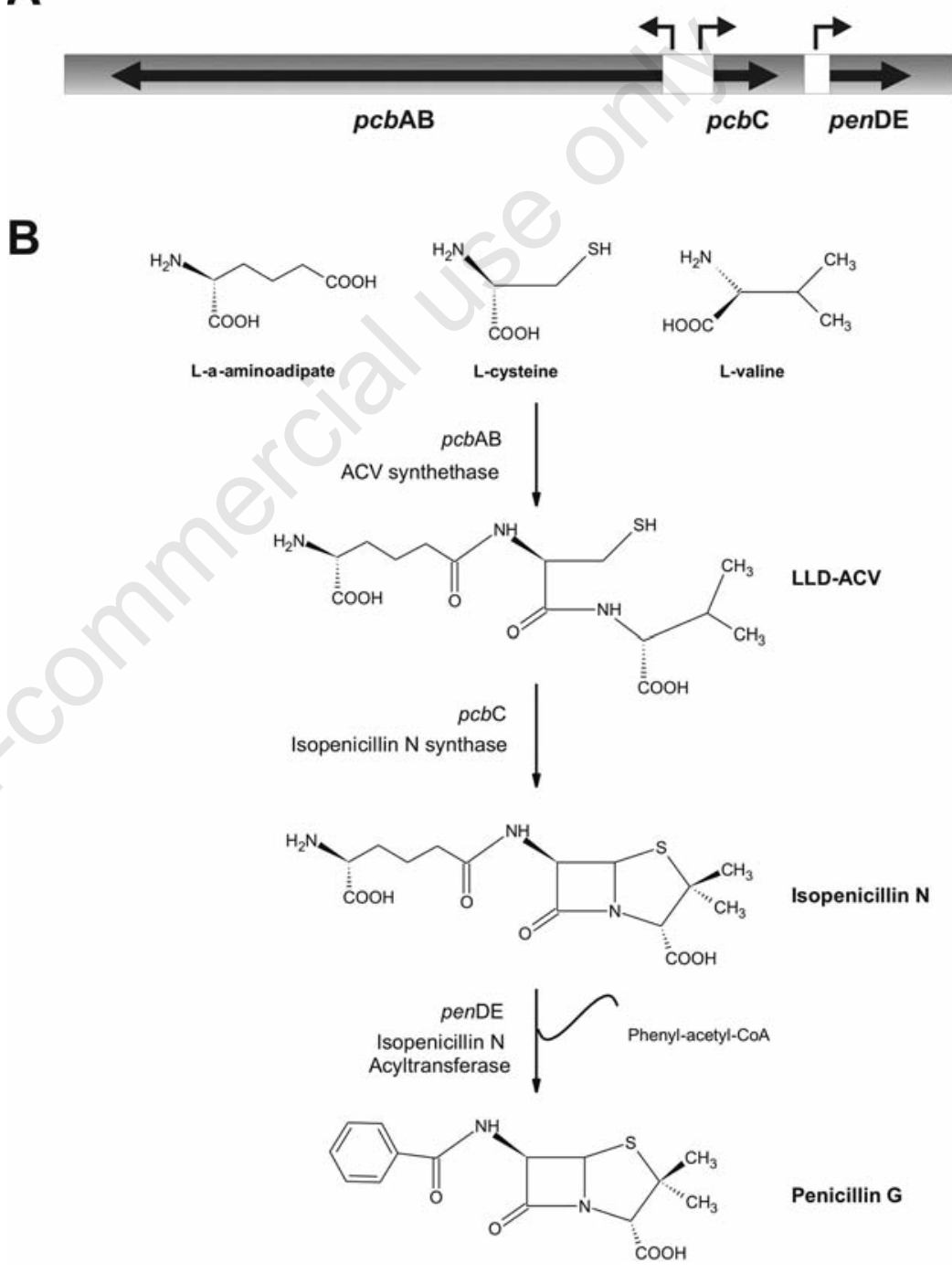

Figure 1. Biosynthesis of the $\beta$-lactam penicillin G. (A) Penicillin gene cluster in the $P$. chrysogenum. The $p c b A B, p c b C$ and penE enconde ACV synthetase, IPN synthase, and IPN acyltransferase, respectively. The black arrows indicate the orientation of the genes. The bidirectional $p c b A B-p c b C$ promoter region and penE promoter are indicated with boxes. (B) Penicillin biosynthetic pathway from the three amino acids precursors. The schematic representation shows the genes and proteins involved in different steps of the biosynthesis of penicillin G. LLD-ACV, $\alpha$-L-aminoadipyl-L-cysteinyl-D-valine; IPN, isopenicillin $\mathbf{N}$. 
Biosystems, USA). Quantitative PCR reactions were performed in a $20 \mu \mathrm{L}$ reaction mixture containing 1X SYBR Green Master Mix (Exiqon, Denmark), $0.2 \mu \mathrm{M}$ each of the forward and reverse reaction primers, and diluted first-strand cDNA. Primer pairs used for quantitative real time PCR reactions were designed using PRIMER3-plus software ${ }^{18}$ from the known sequences for the penicillin biosynthetic genes $p c b A B, p c b C$ and $p e n D E$ genes, and for the constitutively expressed reference gapdh gene from $P$. chrysogenum. The PCR conditions were $95^{\circ} \mathrm{C}$ for $10 \mathrm{~min}$, followed by 40 cycles of $95^{\circ} \mathrm{C}$ for $15 \mathrm{sec}$ and $60^{\circ} \mathrm{C}$ for $1 \mathrm{~min}$. Each PCR reaction was run in triplicate.

For the $p c b A B$ gene, the forward primer 5'TGTGCAGGCCAAGGTAAAC-3' corresponding to nucleotides 3806 to 3825 relative to the start codon and the reverse primer 5'CCAGTTCAGTCTGGTGCTCA-3' complementary to the nucleotides 3948 to 3967 amplified a 162-bp PCR product. For the penDE gene, the forward primer 5'-CGAAGAAGACGGACGAAGA3 ' corresponding to nucleotides 118 to 137 relative to the start codon and the reverse primer 5'- TGCGGGTATTAAGCATGACA -3' complementary to the nucleotides 249 to 268 amplified a 151-bp PCR product. For the $p c b C$ gene, the forward primer 5'-ACGGCACCAAATTGAGTTTC3 ' corresponding to nucleotides 614 to 633 relative to the start codon and the reverse primer 5'- GGTGATGTGTGCCATGTAGC-3' complementary to the nucleotides 764 to 783 amplified a 170-bp PCR product. For the reference gene gapdh, the forward primer 5'-TCGGTATCGTTGAGGGTCTC-3' corresponding to nucleotides 500 to 519 relative to the start codon and the reverse primer 5'-GGTGGAGGAGGGGATAATGT-3' complementary to the nucleotides 611 to 630 amplified a 131-bp PCR product. The amplification efficiencies of the primers used for $p c b A B, p c b C$, penDE and gapdh genes was tested by the standard curve method. Relative expression values were calculated by the comparative $\Delta \Delta \mathrm{Ct}$ method. $\Delta \mathrm{Ct}$ values were determined for all the transcripts using gapdh as a reference gene transcript and the results normalized against the expression data obtained for each mRNA in the medium containing glucose as a sole carbon source.

A parallel assay was performed in each independent experiment to check the differences in PCR efficiency. This parallel assay was designed for analyzing penicillin gene expression by qPCR from two additional carbon sources (glucose and phenol) obtained from the same preinoculum.

\section{Antibiotic production}

Penicillin was estimated by bioassay using the agar-diffusion method with Micrococcus luteus ATCC 9341 (kindly provided by Prof. JF Martín, León University) as an indicator strain as previously described. ${ }^{19}$

\section{Phenol and phenolic compound concentrations}

Phenol, catechol, resorcinol and hydroquinone concentrations were quantified by High Performance Liquid Chromatography (L7100 LaChrom HPLC System, Merck), equipped with a quaternary pump system, and L-7400 UV detector according to a previously published method..$^{17}$ Aromatic compounds were separated and concentrations estimated within $10 \mathrm{~min}$.

\section{Results}

\section{Effect of phenol and phenolic compounds in the expression level of penicillin biosynthetic cluster}

The gene expression levels of penicillin biosynthetic cluster of $P$. chrysogenum var. halophenolicum when grown in mineral medium with $2 \% \mathrm{NaCl}$ and supplemented with glucose, phenol, catechol, hydroquinone and resorcinol as the sole carbon source were analyzed by qPCR. The results of the expression of $p c b A B, p c b C$ and $p e n D E$ genes, expressed as normalized quantities for each gene relative to the glucose sample, are shown in Figure 2. From this figure, it can be seen that despite the relative abundance of each transcript, differences were observed in samples depending on carbon source and culture time, particularly at $45 \mathrm{~h}$. The expression level of penicillin biosynthetic cluster increased for all the carbon sources tested compared to the glucose batch. This increment in $p c b A B, p c b C$ and penDE gene expression was at least a 100 -fold in phenol and phenolic coumponds batches compared to glucose batch.

The expression of $p c b A B$ was higher in batch cultures supplemented with phenol as the sole carbon source; meanwhile, the expression of $p c b A B$ was not significantly affected by phenolic compound. Maximal induction was observed for the $p c b C$ gene, particularly in the case of catechol at $45 \mathrm{~h}$ of cultivation. Expression of $p c b A B, p c b C$ and $p e n D E$ were similar for catechol and hydroquinone.

\section{Effect of salt, phenol and phenolic} compounds on the expression level of penicillin biosynthetic cluster

To examine the expression level of penicillin biosynthetic cluster when $P$. chrysogenum var. halophenolicum is grown under osmotic stress, batch cultures of fungus in mineral medium with $5.9 \% \mathrm{NaCl}$ and supplemented with glucose, phenol, catechol, hydroquinone and resorcinol as the sole carbon source were performed and the expression levels of $p c b A B$, $p c b C$ and $p e n D E$ were analyzed by $\mathrm{qPCR}$. Figure 3 shows the results of $p c b A B, p c b C$ and penDE gene expression after the normalization of quantities for each gene relative to the glucose sample. The mRNA levels from $p c b A B$, $p c b C$ and penDE were reduced by the presence of $5.9 \%$ of sodium chloride. Expression of $p c b A B$ and penDE was lower when fungus was grown with phenol and phenolic compounds than in a glucose batch. Approximately 2 times the $p c b C$ expression was obtained in the phe-
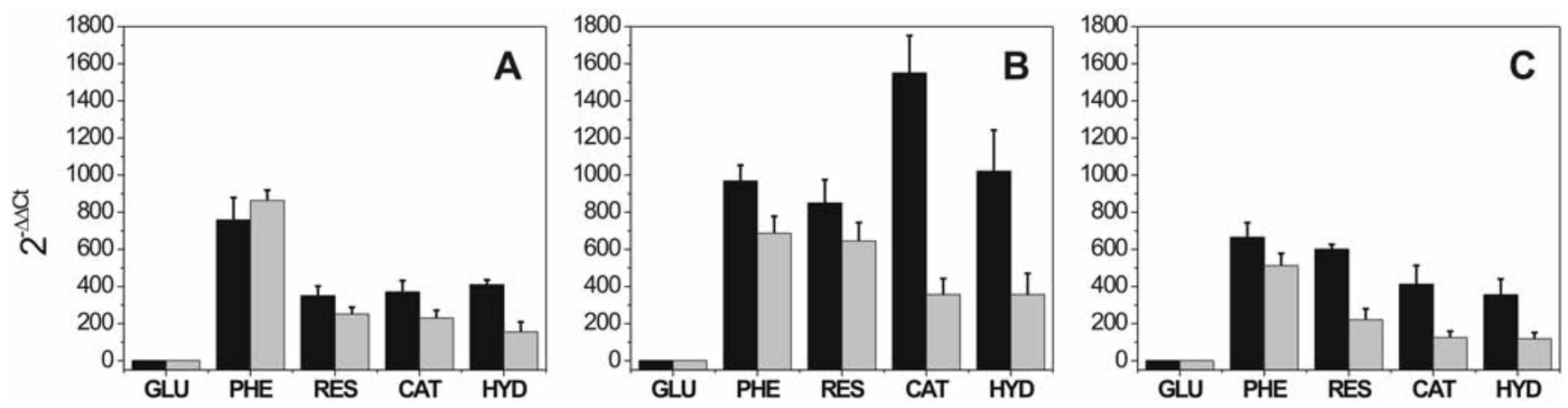

Figure 2. Relative expression values for $p c b A B, p c b C$ and $p e n D E$ messenger RNAs quantified by qPCR in the presence of phenol (PHE), resorcinol (RES), catechol (CAT) and hydroquinone (HYD) in culture with $2 \% \mathrm{NaCl}$ at two different culture times (45 and $68 \mathrm{~h}$ ). Results were normalized against the expression values obtained in the same conditions using glucose (GLU) as the sole carbon source. (A) $p c b A B,(\mathrm{~B}) p c b C$ and $(\mathrm{C})$ penDE. Data correspond to three biological replicates performed in triplicate. 

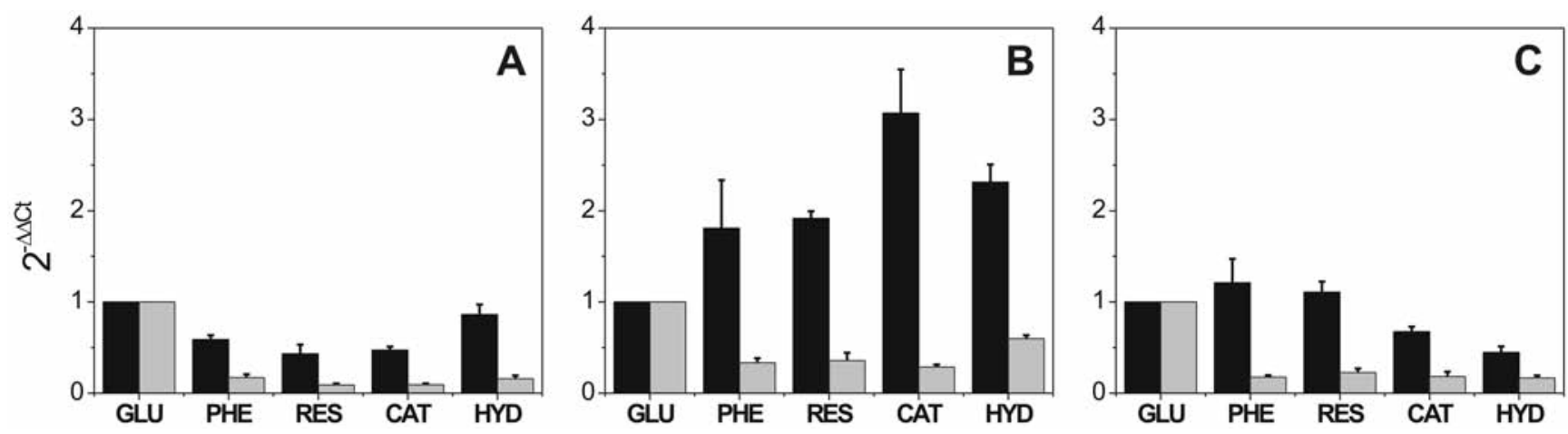

Figure 3. Relative expression values for $p c b A B, p c b C$ and $p e n D E$ messenger RNAs quantified by qPCR in high saline condition (5.9\% $\mathrm{NaCl}$ ) and in the presence of phenol (PHE), resorcinol (RES), catechol (CAT) and hydroquinone (HYQ) at two different culture times (45 and $68 \mathrm{~h}$ ). Results were normalized against the expression values obtained in the same conditions using glucose (GLU) as the sole carbon source. (A) $p c b A B,(\mathrm{~B}) p c b C$ and $(\mathrm{C})$ penDE. Data correspond to three biological replicates performed in triplicate.

nol and phenolic compound batches compared to the glucose batch at $48 \mathrm{~h}$. These results suggest that the salt had a negative effect on the expression of penicillin biosynthetic cluster.

\section{Phenol and phenolic compounds} are used with different efficiency by the $P$. chrysogenum var.

\section{halophenolicum strain}

To determine the ability of $P$. chrysogenum var. halophenolicum to degrade phenol and phenolic compounds under optimal saline conditions $(2 \% \mathrm{NaCl})$ and osmotic stress (5.9\% $\mathrm{NaCl}$ ), cultures with $2.67 \mathrm{mM}$ of phenol, catechol, hydroquinone and resorcinol were performed at two saline concentrations. The degradation of phenol and phenolic compounds presented different rates depending on the salt concentration and aromatic compound tested (Figure 4). When the fungus was grown with phenol, catechol and hydroquinone with $2 \% \mathrm{NaCl}$ after $45 \mathrm{~h}$ of cultivation none of these aromatic compounds were detected in the samples analyzed by HPLC. Meanwhile, for the same culture time $(45 \mathrm{~h})$, approximately $38.5 \%, 71.0 \%$ and $83.1 \%$ of phenol, hydroquinone and catechol were degraded by fungus, respectively, in samples from culture performed with $5.9 \%$ of salt. Resorcinol was the aromatic compound that was less efficiently degraded by $P$. chrysogenum var. halophenolicum. The percentage of catechol and hydroquinone removal was higher than phenol and resorcinol at both times of cultivation.

These findings suggest that sodium chloride concentration regulated the phenol and phenolic compound degradation of $P$. chrysogenum var. halophenolicum. One explanation for the decrease of phenol and phenolic compound uptake was osmotic balance which would have resulted in a decreased of the biodegradation rate in high salt concentration cultures.
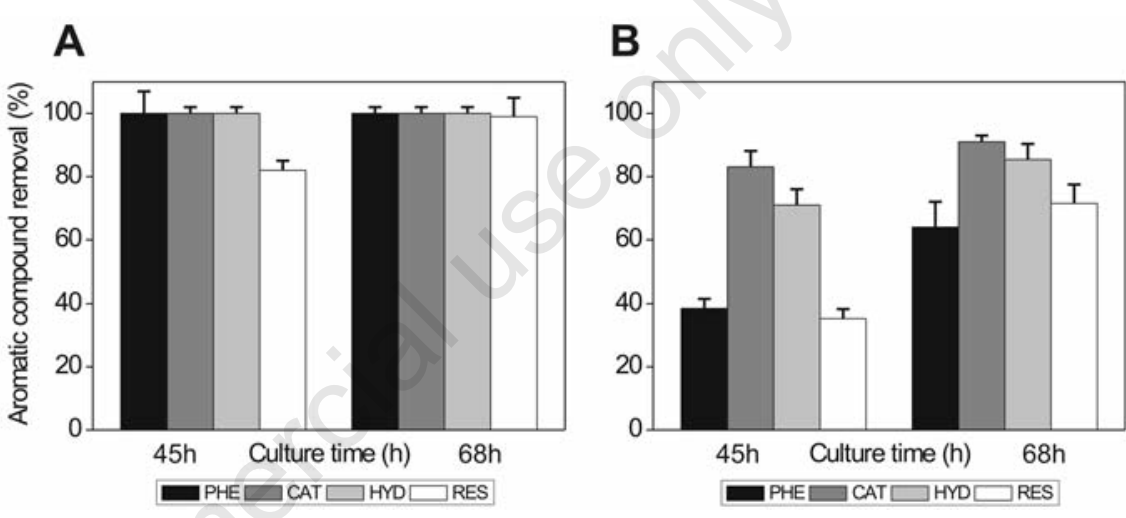

Figure 4. Degradation of $2.67 \mathrm{mM}$ of phenol and phenolic compound as the sole carbon source by $P$. chrysogenum var. halophenolicum in two different saline conditions. (A) $2 \%$ $\mathrm{NaCl}$ and (B) $5.9 \% \mathrm{NaCl}$. PHE, phenol; RES, resorcinol; CAT, catechol; HYD, hydroquinone. Error bars represent the standard deviation of three replicates.

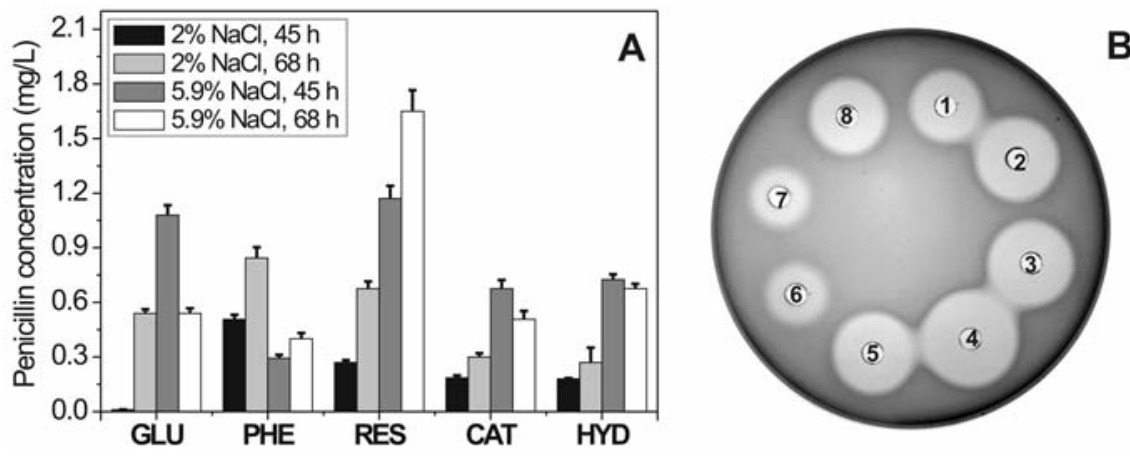

Figure 5. Antibiotic production by $P$. chrysogenum var. halophenolicum in mineral medium supplemented with glucose (GLU), phenol (PHE), resorcinol (RES), catechol (CAT) and hydroquinone (HYD) at two saline concentrations (2\% and $5.9 \% \mathrm{NaCl})$. (A) Penicillin production at two culture times $(45 \mathrm{~h}$ and $68 \mathrm{~h})$. (B) Bioassay using diluted culture filtrates (dilution 1:2) obtained at $68 \mathrm{~h}$ in cultures with phenol and $5.9 \% \mathrm{NaCl}(1)$, catechol and $5.9 \% \mathrm{NaCl}(2)$, hydroquinone and $5.9 \% \mathrm{NaCl}(3)$, resorcinol and $5.9 \%$ $\mathrm{NaCl}(4)$, phenol and $2 \% \mathrm{NaCl}(5)$, catechol and $2 \% \mathrm{NaCl}(6)$, hydroquinone and $2 \%$ $\mathrm{NaCl}(7)$, resorcinol and $2 \% \mathrm{NaCl}(8)$. 


\section{Effect of phenol and phenolic compounds on penicillin \\ biosynthesis}

Penicillin production by $P$. chrysogenum var. halophenolicum with phenol, catechol, hydroquinone or resorcinol was quantified in batch cultures at $45 \mathrm{~h}$ and $68 \mathrm{~h}$. Differences in $\beta$-lactam antibiotic production are clearly seen in mineral medium supplemented with $2 \%$ and $5.9 \% \mathrm{NaCl}$ (Figure 5). Moreover, differences were observed in the penicillin pattern of phenolic compound cultures. Penicillin production was considerably higher in the cultures with $5.9 \%$ of salt at $45 \mathrm{~h}$. Antibiotic production in the resorcinol batch was higher at both times in the cultures supplemented with 5.9\% $\mathrm{NaCl}$, whereas in the cultures with lower salt, penicillin production was higher in the phenol cultures. No difference was observed in penicillin production of catechol and hydroquinone cultures.

The onset of penicillin biosynthesis in the glucose cultures at $2 \%$ of salt took place after $36 \mathrm{~h}$, although penicillin production was very small after $45 \mathrm{~h}$. However, the penicillin production rate was considerably higher in the cultures with glucose as the sole carbon source supplemented with $5.9 \%$ of $\mathrm{NaCl}$.

Penicillin yields of $P$. chrysogenum var. halophenolicum in the cultures containing resorcinol resulted in an antibiotic increase of about $300 \%$ with respect to the glucose at $68 \mathrm{~h}$.

\section{Discussion}

The $p c b A B, p c b C$ and $p e n D E$ are the three penicillin biosynthesis genes that are present in a few evolutionarily related fungal species. ${ }^{5,20}$ The conserved arrangement of the penicillin biosynthesis cluster in different species is one of the most important features that enable the coordinated regulation of the three genes in $\beta$-lactam antibiotic pathway. ${ }^{21} P$. chrysogenum var. halophenolicum (previously known as CLONA2) showed an overall high conservation degree between its penicillin gene cluster and the NRRL1951 (wild-type strain) or Wis54-1255 (an improved, moderate penicillin producer) strains. ${ }^{15}$ Penicillin production is affected by some aspects of primary metabolism related to sugar catabolic pathways and energy balance. ${ }^{10,22}$ Several groups have described that transcription of the genes encoding for the enzymes in the penicillin biosynthesis pathway is repressed by glu-

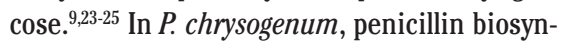
thesis is repressed by glucose preventing expression of the $p c b A B, p c b C$ and $p e n D E$ genes. ${ }^{25}$ According to published results, glucose induced not only higher rates of respiration but also greater energy production than lactose as carbon source in $P$. chrysogenum NRRL 1915 strain. ${ }^{10}$ The presence of glucose caused a delay in penicillin production in the culture condition with $2 \%$ of salt. This result is in agreement with that reported about the effect of glucose on penicillin biosynthetic cluster. As shown in this article, lower levels of $p c b A B$ and penDE gene expression were observed in phenol, catechol, hydroquinone and resorcinol containing medium with $5.9 \%$ $\mathrm{NaCl}$ compared to glucose condition, indicating that these genes are repressed by phenol and phenolic compounds. Meanwhile, penicillin production increased when the fungus was cultivated in a high saline concentration with catechol, hydroquinone and resorcinol as the carbon source. In other words, the expression level of $p c b A B$ and penDE does not seem to be a relevant factor for antibiotic biosynthesis. Douma et al. ${ }^{24}$ reported that the control of penicillin biosynthesis is exerted by a single rate-limiting enzyme: the isopenicillin $\mathrm{N}$ synthase encoded by the $p c b C$ gene. Meanwhile, other groups report that the rate limiting step reside either at $\delta$-(L- $\alpha$-aminoadipyl)-L-cysteinyl-D-valine synthetase or at isopenicillin $\mathrm{N}$ synthase. ${ }^{26,27}$ In this present study, the $p c b C$ gene was among those showing higher expression levels. Indeed the expression of $p c b C$ was at least 200 times higher in phenol and phenolic compounds containing medium than in cultures with glucose and $2 \% \mathrm{NaCl}$. The $p c b C$ gene expressed differentially in catechol containing medium; expression levels 1,500 times higher than in glucose containing medium was observed at $45 \mathrm{~h}$ of cultivation, while an expression of 300 times was quantified at $68 \mathrm{~h}$. The fermentation times for this study, $45 \mathrm{~h}$ and $68 \mathrm{~h}$, were chosen in consideration of the fact that most secondary genes tend to show patterns of late expression and the phenol and phenolic compound removal rates. Low yields of penicillin were observed compared with the expression level of the antibiotic biosynthetic genes. This result is not surprising because in gene dosage studies it is frequently observed that a high increase in gene copy number does not correspond with a parallel increment in enzyme activity. ${ }^{28}$ On the other hand, other phenomena besides genes expression could be responsible for the antibiotic production. Nasutation et al. ${ }^{29}$ have shown from a penicillin producing strain in glucose-limited chemostat cultures that the penicillin production flux appears to be mostly influenced by cystein, one of the three precursor amino acids, and by energy and redox cofactors. The relation between the low penicillin production flux and the low ATP and NADPH levels could be a possible explanation for the present results. Furthermore, it was reported that penicillin biosynthesis was accompanied by the consumption of a surprising large amount of ATP. ${ }^{30}$ Another hypothesis for the present results could be related to toxicity. As we know, very high amounts of hydrophilic penicillins could exert toxic effects on eukaryotic cells caused by the lipophilic interactions with intracellular membranes and plasma membrane. ${ }^{2,31}$ Since it has been shown that $P$. chrysogenum var. halophenolicum strain produced non-aromatic natural penicillins rather than benzylpenicillin, this hypothesis does not seem to be the principal reason for the low penicillin yields. ${ }^{15}$ However, we cannot exclude the possibility that non-aromatic natural penicillins might not be actively pumped out of the cells in $P$. chrysogenum var. halophenolicum.

There was no evident relationship between the expression levels of penicillin biosynthetic genes and yields of penicillin. Meanwhile, the presence of phenol and phenolic compounds seems to influence antibiotic production. Moreover, in catechol and hydroquinone cultures, where the phenolic compounds were more efficiently removed by $P$. chrysogenum var. halophenolicum, the production of penicillin was lower than in fermentations containing phenol and resorcinol. Despite the previous observations that described the absence of the metabolic control in $P$. chrysogenum, ${ }^{32}$ meaning that the carbon source had no effect on the penicillin production rate, we found a relationship between carbon metabolism and antibiotic biosynthesis. Interestingly, a positive relationship was observed in media with phenol under $2 \% \mathrm{NaCl}$ between the penicillin titers, expression levels of penicillin biosynthetic genes and carbon metabolism. In this case, a positive relationship was found between the efficiency of aromatic removal and antibiotic production. This fact could be explained by the mimicking effect between phenol and phenylacetic acid, a compound that is known to stimulate the penicillin biosynthetic pathway as a precursor of benzylpenicillin. ${ }^{15}$ In the present study, we also observed that high concentrations of salt increased penicillin production yields. Since in a previous work it was reported that $2 \%$ of $\mathrm{NaCl}$ is the optimum concentration of salt for P. chrysogenum var. halophenolicum, this result suggests that the antibiotic secretion could be a defence mechanism against osmotic stress.

In summary, today, biological treatment is still an interesting process to reduce the negative impact of toxic compounds. The ability of P. chrysogenum var. halophenolicum to degrade catechol and hydroquinone was shown in conditions of $2 \%$ and $5.9 \%$ salt. The secretion of secondary metabolites with antibacterial activity is a useful natural tool for the microorganisms that produce them. ${ }^{2,33}$ The present results have shown that $P$. chrysogenum var. halophenolicum produced $\beta$-lactam antibiotic in phenol and phenolic compounds containing medium with $2 \%$ and $5.9 \%$ of salt. These results support the hypothesis that phe- 
nol, phenolic compounds and high concentrations of salt could behave like stress factors triggering secondary metabolism in $P$. chrysogenum var. halophenolicum, and resulting in higher $\beta$-lactam antibiotic production. This could constitute an advantage for $P$. chrysogenum var. halophenolicum in wastewater treatment systems, since the production of a secondary metabolite such as penicillin could make it possible to establish the fungal strain, exerting a positive selection pressure over the endogenous native microbial population.

\section{References}

1. Elander RP. Industrial production of betalactam antibiotics. Appl Microbiol Biotechnol 2003;61:385-92.

2. Garcia-Estrada C, Vaca I, Lamas-Maceiras $\mathrm{M}$, Martin JF. In vivo transport of the intermediates of the penicillin biosynthetic pathway in tailored strains of Penicillium chrysogenum. Appl Microbiol Biotechnol 2007;76:169-82.

3. Martin MF, Liras P. Organization and expression of genes involved in the biosynthesis of antibiotics and other secondary metabolites. Annu Rev Microbiol 1989;43:173-206.

4. Newbert RW, Barton B, Greaves P, Harper J, Turner G. Analysis of a commercially improved Penicillium chrysogenum strain series: involvement of recombinogenic regions in amplification and deletion of the penicillin biosynthesis gene cluster. J Ind Microbiol Biotechnol 1997;19:18-27.

5. Martin JF. Molecular control of expression of penicillin biosynthesis genes in fungi: regulatory proteins interact with a bidirectional promoter region. J Bacteriol 2000; 182:2355-62.

6. Diez B, Gutierrez S, Barredo JL, van Solingen P, van der Voort LH, Martin JF. The cluster of penicillin biosynthetic genes. Identification and characterization of the $p c b A B$ gene encoding the alphaaminoadipyl-cysteinyl-valine synthetase and linkage to the $p c b C$ and $p e n D E$ genes. J Biol Chem 1990;265:16358-65.

7. Ramos FR, Lopez-Nieto MJ, Martin JF. Isopenicillin $\mathrm{N}$ synthetase of Penicillium chrysogenum, an enzyme that converts delta-(L-alpha-aminoadipyl)-L-cysteinylD-valine to isopenicillin N. Antimicrob Agents Chemother 1985;27:380-7.

8. Muller WH, van der Krift TP, Krouwer AJ, Wosten HA, van der Vort LH, Smaal EB, et al. Localization of the pathway of the penicillin biosynthesis in Penicillium chrysogenum. EMBO J 1991;10:489-95.

9. Revilla G, Lopez-Nieto MJ, Luengo JM, Martin JF. Carbon catabolite repression of penicillin biosynthesis by Penicillium chrysogenum. J Antibiot 1984;37:781-9.

10. Castillo NI, Fierro F, Gutierrez S, Martin JF. Genome-wide analysis of differentially expressed genes from Penicillium chrysogenum grown with a repressing or a nonrepressing carbon source. Curr Genet 2006;49:85-96.

11. Alva VA, Peyton BM. Phenol and catechol biodegradation by the haloalkaliphile Halomonas campisalis: influence of $\mathrm{pH}$ and salinity. Environ Sci Technol 2003; 37:4397-402.

12. Bastos AE, Moon DH, Rossi A, Trevors JT, Tsai SM. Salt-tolerant phenol-degrading microorganisms isolated from Amazonian soil samples. Arch Microbiol 2000;174:34652.

13. Hinteregger C, Streichsbier F. Halomonas sp., a moderately halophilic strain, for biotreatment of saline phenolic wastewater. Biotechnol Lett 1997;19:1099-102.

14. Peyton BM, Wilson T, Yonge DR. Kinetics of phenol biodegradation in high salt solutions. Water Res 2002;36:4811-20.

15. Leitão AL, Garcia-Estrada C, Ullan RV, Guedes SF, Martin-Jimenez, Mendes B, et al. Penicillium chrysogenum var. halophenolicum, a new halotolerant strain with potential in the remediation of aromatic compounds in high salt environments. Microbiol Res 2012;167:79-89.

16. Guedes SF, Mendes B, Leitao AL. Resorcinol degradation by a Penicillium chrysogenum strain under osmotic stress: mono and binary substrate matrices with phenol. Biodegradation 2011;22:409-19.

17. Leitão AL, Duarte MP, Oliveira JS. Degradation of phenol by a halotolerant strain Penicillium chrysogenum. Int Biodeter Biodeg 2007;59:220-5.

18. Untergasser A, Nijveen H, Rao X, Bisseling T, Geurts R, Leunissen JA. Primer3Plus, an enhanced web interface to Primer3. Nucleic Acids Res 2007;35:W71-4.

19. Garcia-Estrada C, Vaca I, Fierro F, Sjollema $\mathrm{K}$, Veenhuis M, Martin JF. The unprocessed preprotein form IATC103S of the isopenicillin $\mathrm{N}$ acyltransferase is transported inside peroxisomes and regulates its self-processing. Fungal Genet Biol 2008;45:1043-52.

20. Aharonowitz Y, Cohen G, Martin JF. Penicillin and cephalosporin biosynthetic genes: structure, organization, regulation, and evolution. Annu Rev Microbiol 1992; 46:461-95.

21. Martin JF, Gutierrez S. Genes for beta-lactam antibiotic biosynthesis. Antonie Van Leeuwenhoek 1995;67:181-200.

22. Thykaer J, Nielsen J. Metabolic engineering of beta-lactam production. Metab Eng 2003;5:56-69.

23. Brakhage AA, Browne P, Turner G.
Regulation of Aspergillus nidulans penicillin biosynthesis and penicillin biosynthesis genes acvA and ipnA by glucose. J Bacteriol 1992;174:3789-99.

24. Douma RD, Verheijen PJ, de Laat WT, Heijnen JJ, van Gulik WM. Dynamic gene expression regulation model for growth and penicillin production in Penicillium chrysogenum. Biotechnol Bioeng 2010; 106:608-18.

25. Gutierrez S, Marcos AT, Casqueiro J, Kosalkova K, Fernandez FJ, Velasco J, et al. Transcription of the $p c b A B, p c b C$ and penDE genes of Penicillium chrysogenum AS-P-78 is repressed by glucose and the repression is not reversed by alkaline $\mathrm{pHs}$. Microbiology 1999;145:317-24.

26. Nielsen J, Jorgensen HS. Metabolic control analysis of the penicillin biosynthetic pathway in a high-yielding strain of Penicillium chrysogenum. Biotechnol Prog 1995;11:299-305.

27. Revilla G, Ramos FR, Lopez-Nieto MJ, Alvarez E, Martin JF. Glucose represses formation of delta-(L-alpha-aminoadipyl)-Lcysteinyl-D-valine and isopenicillin $\mathrm{N}$ synthase but not penicillin acyltransferase in Penicillium chrysogenum. J Bacteriol 1986;168:947-52.

28. Chary VK, de la Fuente JL, Leitão AL, Liras P, Martin JF. Overexpression of the lat gene in Nocardia lactamdurans from strong heterologous promoters results in very high levels of lysine-6-aminotransferase and up to two-fold increase in cephamycin C production. Appl Microbiol Biotechnol 2000;53:282-8.

29. Nasution U, van Gulik WM, Ras C, Proell A, Heijnen JJ. A metabolome study of the steady-state relation between central metabolism, amino acid biosynthesis and penicillin production in Penicillium chrysogenum. Metab Eng 2008;10:10-23.

30. van Gulik WM, Antoniewicz MR, deLaat WT, Vinke JL, Heijnen JJ. Energetics of growth and penicillin production in a highproducing strain of Penicillium chrysogenum. Biotechnol Bioeng 2001;72:185-93.

31. Sikkema J, de Bont JA, Poolman B. Mechanisms of membrane toxicity of hydrocarbons. Microbiol Rev 1995;59:20122.

32. van Gulik WM, de Laat WT, Vinke JL, Heijnen JJ. Application of metabolic flux analysis for the identification of metabolic bottlenecks in the biosynthesis of penicillin-G. Biotechnol Bioeng 2000;68:60218.

33. Martin JF, Casqueiro J, Liras P. Secretion systems for secondary metabolites: how producer cells send out messages of intercellular communication. Curr Opin Microbiol 2005;8:282-93. 